\title{
Shape-induced phase transition of domain patterns in ferroelectric platelets
}

\author{
A. Schilling, ${ }^{1}$ S. Prosandeev, ${ }^{2}$ R. G. P. McQuaid, ${ }^{1}$ L. Bellaiche, ${ }^{2}$ J. F. Scott,${ }^{3}$ and J. M. Gregg ${ }^{1, *}$ \\ ${ }^{1}$ Centre for Nanostructured Media, School of Maths and Physics, Queen's University Belfast, Belfast, BT7 1NN, UK \\ ${ }^{2}$ Department of Physics and The Arkansas Institute for Nanoscience and Engineering, University of Arkansas, \\ Fayetteville, Arkansas 72701, USA \\ ${ }^{3}$ Department of Physics, Cavendish Laboratory, University of Cambridge, J. J. Thompson Avenue, Cambridge, CB3 OHE, UK
}

(Received 24 May 2011; revised manuscript received 7 July 2011; published 23 August 2011)

\begin{abstract}
Using a combination of experimental and computational techniques, changes in the domain structures seen in freestanding single-crystal platelets of $\mathrm{BaTiO}_{3}$ have been described in terms of a second-order phase transition. The transition is driven by the change in the length-to-width ratio of the platelet sidewalls and results in a symmetry breaking of a complex, quadrant domain pattern. The phenomenon can be described by a Landau formalism in which (1) the order parameter is not the polarization but rather is the degree to which the domain pattern becomes off-centered, and (2) the shape anisotropy of the platelet substitutes for temperature in the conventional Landau expansion as the controlling thermodynamic variable. Bistability, in terms of the direction in which the domain pattern moves off center, coupled with the spontaneous macroscopic polarization and toroidal moment that result from this off-centering, prompt the possibility of a new form of memory storage.
\end{abstract}

DOI: 10.1103/PhysRevB.84.064110

PACS number(s): 64.70.Nd, 64.60.Bd, 77.22.Ej, 77.80.Dj

\section{INTRODUCTION}

As is the case in many materials systems, the variety of physical characteristics displayed by ferroelectrics is dramatically enriched by the occurrence of phase transitions. Indeed, for most ferroelectrics, the development of the field-reversible spontaneous polarization, which is the defining characteristic for this family of materials, ${ }^{1,2}$ is itself the product of a phase transition: in general, a high-temperature state spontaneously changes on cooling to a low-temperature state in which electrical dipoles form and cooperatively align. In bulk, most aspects of these phase transitions can be described and understood using simple phenomenological Landau theories,${ }^{3-5}$ in which the thermodynamic quantity controlling the transition is the temperature, while the order parameter is the polarization. In small-scale ferroelectrics, however, the proximity of surfaces and reductions in material volume can allow unusual order parameters to appear, such as the toroidal moment of the polarization rather than the polarization itself; ${ }^{6-8}$ moreover, the range of thermodynamic variables responsible for driving the phase transitions could, in principle, increase to include aspects specifically related to reduced size. Phase transition mapping in small-scale objects is therefore of significant interest. ${ }^{9-11}$

In this paper, we present observations made on the domain arrangements found in mesoscale rectangular platelets of $\mathrm{BaTiO}_{3}$. We note, through both experiment and simulation, the common occurrence of sets of $90^{\circ}$ stripe domains ${ }^{12-14}$ which form into four distinct blocks or quadrants surrounding a single quadrant core (generating patterns similar to those seen previously in bulk by Forsbergh ${ }^{15}$ ). Surprisingly, the position of this quadrant core depends critically upon the aspect ratio of the sidewall dimensions of the platelets. Analysis of the energy as a function of core position, implied by our computational simulations, shows that the migration of the quadrant core (from the geometric center in square platelets to progressively greater levels of off-centering in more elongated rectangles) can be described as a shape-induced phase transition. This phase transition involves complex dipolar configurations, the symmetry of which is critically related to shape anisotropy. A spontaneous macroscopic polarization and electric toroidal moment also develop during the transition.

\section{EXPERIMENTAL METHODOLOGY}

In order to make experimental observations of the domain patterns present in $\mathrm{BaTiO}_{3}$ platelets, the following sample preparation methodology was used: thin single crystal lamellae (with dimensions of $10 \times 15 \mu \mathrm{m}^{2}$ and $\sim 150-200 \mathrm{~nm}$ in thickness) were milled out from commercially obtained bulk $\mathrm{BaTiO}_{3}$ single crystals, using an FEI 200 TEM focused ion beam (FIB) microscope. All lamellae were cut with $\langle 100\rangle_{\mathrm{pc}(\mathrm{pseudocubic})}$ approximately perpendicular to the lamellar plane. They were then lifted out (using a sharpened glass needle and micromanipulator) onto mesoporous carbon-coated $\mathrm{Cu}$ grids. Subsequent FIB patterning into the face of the lamellae produced isolated platelets with edges machined approximately parallel to the in-plane $\langle 010\rangle_{\mathrm{pc}}$ directions. Dark field scanning transmission electron microscopy (STEM) using an FEI Tecnai F20 and high angular annular dark field (HAADF) detector was then employed for imaging the domains. All samples were heated to above the Curie temperature in the vacuum environment of the TEM column by focusing down the electron beam and noting the disappearance of domain contrast. Domains that formed on cooling through the Curie temperature (on defocusing the electron beam) were then examined. While previous work had shown that surface damage caused by FIB milling could be repaired by thermal annealing, ${ }^{16}$ this could not be done in the current study, as the structural integrity of the mesoporous carbon grids was lost after heating to above $\sim 300{ }^{\circ} \mathrm{C}$. Thus an amorphous surface layer $\sim 20 \mathrm{~nm}$ thick was expected to surround each of the $\mathrm{BaTiO}_{3}$ platelets.

\section{RESULTS AND DISCUSSION}

The micrograph shown in Fig. 1(a) illustrates the domain patterns typically observed. Conventional domain boundaries 


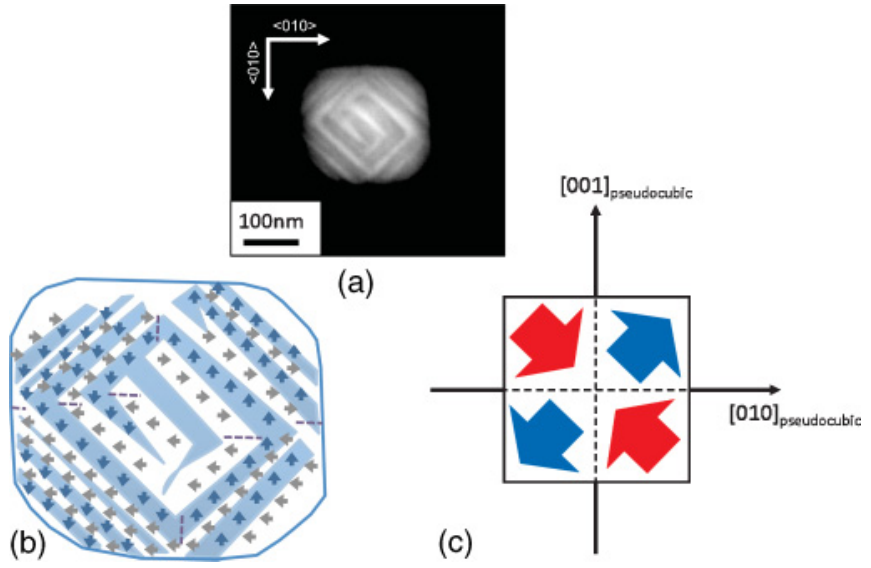

FIG. 1. (Color online) (a) Scanning transmission electron microscopy (STEM) image of a square platelet in which a quadrant arrangement of four blocks of stripe domains can be clearly seen. (b) A schematic illustration of one of the possible local dipole orientations associated with the quadrant structure. In assigning possible dipole directions, it was assumed that the white and dark contrast in the STEM images is associated with the two different in-plane orientations of the [001] tetragonal $c$ axis and that the majority of the simple $90^{\circ}$ and inferred $180^{\circ}$ domain walls are uncharged. Using these assumptions, several possible dipole patterns emerge, but they are degenerate: all result in an overall quadrupole, such as that shown schematically in (c).

between parallel stripe features lie parallel to $\{110\}_{\text {pc }}$ planes, implying that they separate regions in which the polarizations lie at $\sim 90^{\circ}$ to each other, in the plane of the micrograph. They are thus classical in-plane $90^{\circ}$ domains, or a-a domains. Figure 1(a) also clearly shows the manner in which four distinct blocks of these a-a domains are arranged into quadrants. A rationalization for the existence of these quadrants is not straightforward. Nevertheless, given the similarities between the quadrants observed here and the flux closure patterns commonly observed in patterned ferromagnetic structures, ${ }^{17}$ an electric field closure assumption is tempting. However, detailed consideration of the net polarization associated with each quadrant [Figs. 1(b) and 1(c)] shows that they are not field-closure structures at all; rather they form quadrupole states, similar to the antivortex topological defects widely recognized in the magnetics community but only recently considered in ferroelectrics. ${ }^{18,19}$ The dipolar directions marked explicitly in Fig. 1(b) have been reconstructed by assuming, firstly, that the majority of the domain walls are uncharged and, secondly, that the white and dark contrast observed corresponds to the two distinct possible orientations of the crystallographic tetragonal [001] direction in the plane of the platelet. There are several ways that dipoles can be assigned using these two assumptions, but all form a resultant structure in which the overall resolved polarization within each quadrant leads to the inferred existence of a global quadrupole [as illustrated in Fig. 1(c)].

Observations made on larger platelets showed quadrantquadrupole structures to be a common microstructural feature (Fig. 2). From Fig. 2, it is evident that each platelet supports a single quadrant-quadrupole and that although the core of this structure resides close to the geometric center in the square

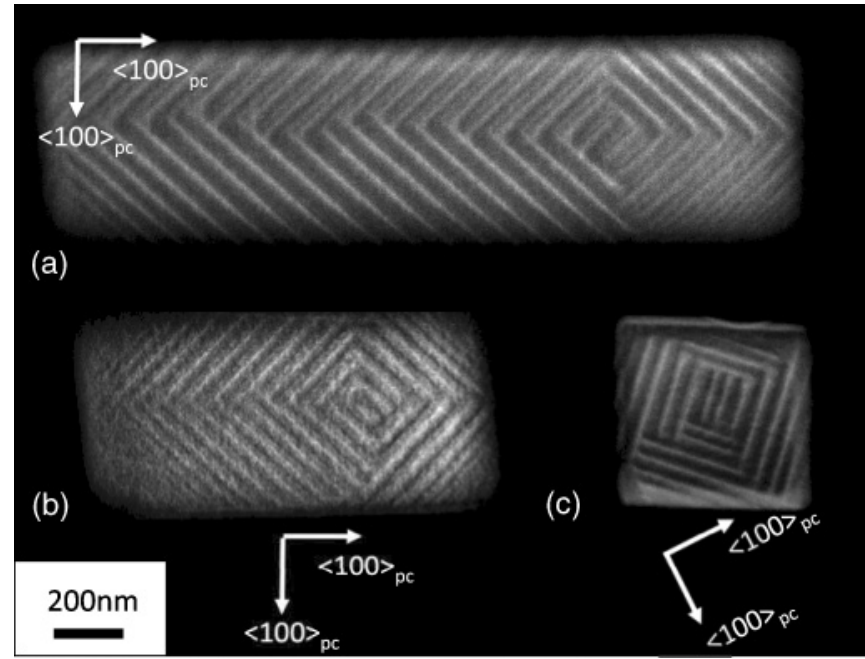

FIG. 2. Scanning transmission electron microscopy (STEM) images of single crystal $\mathrm{BaTiO}_{3}$ platelets taken using a high angle annular dark-field detector (HAADF), in which single well-formed quadrant structures have developed on cooling through the Curie temperature. Aspect ratios of the sidewall lengths of these platelets are (a) $\sim 3: 1$, (b) $\sim 2: 1$, and (c) $\sim 1: 1$.

platelets, it moves distinctly off center in the rectangles. Notably, the degree of off-centering along the length of the platelet [defined by the function $1-(2 x / L)$, where $x$ is the distance from the quadrant core to the nearest end of the platelet, and $L$ is the platelet length] was found to be a distinct function of the aspect ratio of the platelet sidewalls, increasing monotonically as the aspect ratio increased [Fig. 3(a)].

To help rationalize these observations, Monte Carlo computational simulations were employed. A key feature of this modeling was to try to mimic a mesoscopic system for which the degrees of freedom were the polarizations of each
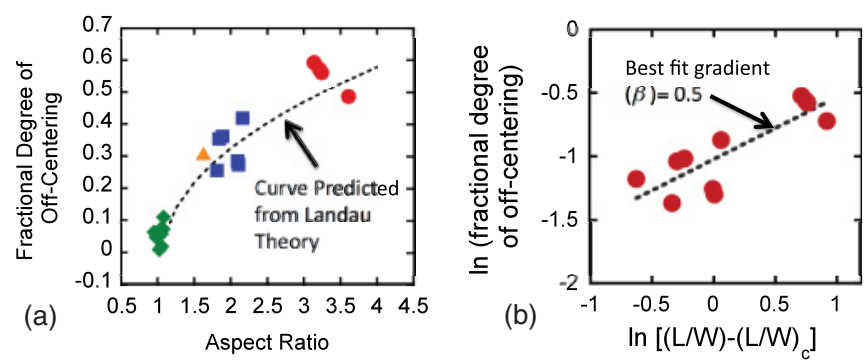

FIG. 3. (Color online) The extent to which quadrant cores migrate to off-centered locations, along the lengths of the $\mathrm{BaTiO}_{3}$ rectangular platelets, was found to progress as a monotonic function of the aspect ratio of the platelet sidewall dimensions (a) [sidewall aspect ratio $\sim 1: 1$ (green filled diamonds), $\sim 2: 1$ (blue filled squares), $\sim 3: 1$ (red filled circles); yellow triangle is a data point from a $2: 1$ platelet whose length was reduced by subsequent focused ion beam milling to create a sidewall aspect ratio of $\sim 3: 2]$. The equilibrium position of the quadrant core predicted by Landau theory is given by the dotted curve in (a). The graph shown in (b) replots the experimental data points in a way in which the critical exponent $(\beta)$ can be readily determined; the value of 0.5 is that classically associated with meanfield treatments of phase transitions. 
(a)

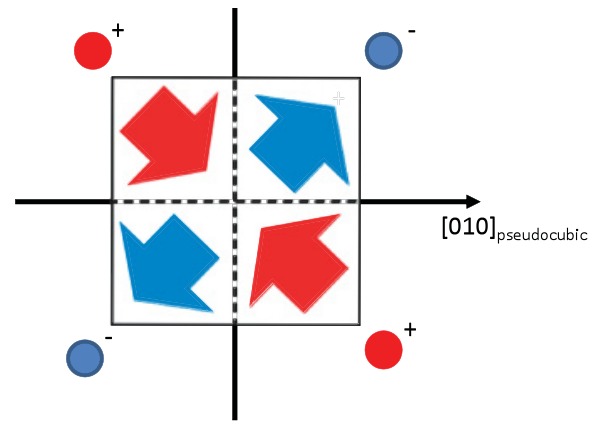

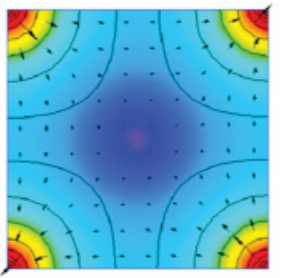

(b)

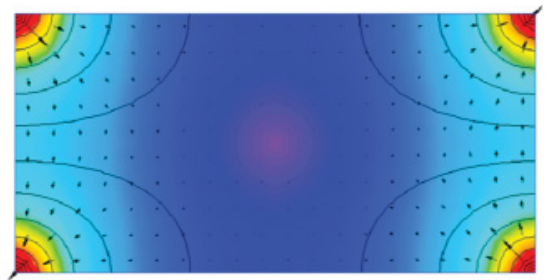

(c)
FIG. 4. (Color online) (a) In setting up the framework for the Monte Carlo simulations, it was assumed that the quadrantquadrupole domain configurations were forming in response to electrostatic boundary conditions that could be effectively recreated by considering point charges at the junctions between platelet sidewalls, creating a quadrupole field. Finite element simulations (using the commercial 'Quickfield' package) for both (b) a square and (c) a rectangular platelet show that the electric field distribution associated with these point charges is always centrosymmetric.

nanometric domain, rather than the electric dipoles centered at each 5-atom primitive cell. The starting point was the effective Hamiltonian constructed for $\mathrm{BaTiO}_{3}$ dots in Ref. 7, but under short-circuit electrical boundary conditions. ${ }^{9}$ This scheme was then modified: the sign of the anharmonic self-energy parameter was reversed (to mimic data at $300 \mathrm{~K}$, at which temperature bulk $\mathrm{BaTiO}_{3}$ is known to be tetragonal rather than rhombohedral), and the dipolar long-range interactions were altered to be governed by nominal ionic charges rather than by the dynamical charges. Similarly, the parameters quantifying the short-range dipolar interactions between firstnearest domains and second-nearest domains were annihilated and enhanced, respectively. Additionally, two energetic terms were added into the total energy of the effective Hamiltonian of Ref. 7. One term [of the form $\alpha \Sigma\left(P_{i, x}^{2} P_{i+1, y}^{2}+P_{i, y}^{2} P_{i+1, x}^{2}\right)$, where $P_{i}$ is the polarization in domain $\left.i\right]$ characterizes biquadratic transverse interactions between polarizations located at nearest-neighbor domains. The other additional term represented the interaction between the set of four charges indicated in Fig. 4(a) (each having the same magnitude as the polarizations of the different domains). These charges were introduced as a pragmatic measure: it was assumed that the quadrant-quadrupole domain configurations were forming in response to specific electrostatic boundary conditions that could be effectively recreated by considering point charges at the junctions between platelet sidewalls [creating a quadrupole field as indicated in Fig. 4(a)]. Importantly, the variation in the magnitude of the electric field associated with this distribution

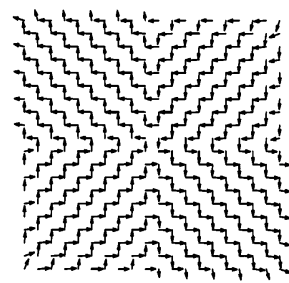

(a)

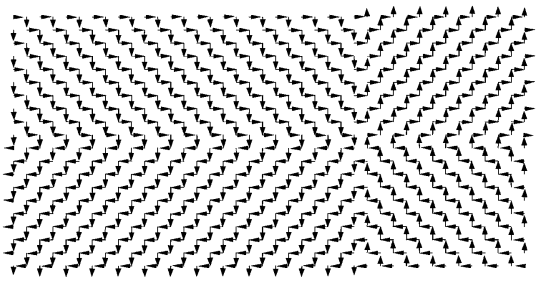

(b)
FIG. 5. While the equilibrium local dipole regions predicted by Monte Carlo simulations do not replicate all features of the experimentally observed domains, it is clear that a change from a quadrant pattern with a centered core position to an off-centered position develops as the platelet sidewall aspect ratio changes.

of charge remains centrosymmetric, irrespective of the aspect ratio of the rectangular platelet [Figs. 4(b) and 4(c)]. Despite this, in general, the equilibrium dipole pattern resulting from Monte Carlo computational simulations breaks the center of symmetry, showing clear quadrant groupings of dipoles in which the quadrant core is distinctly off-centered for all shapes save for the square platelets, mirroring experimental observations (Fig. 5). Monte Carlo simulations also reveal that, as soon as the quadrant core moves off-center, a net polarization and nonzero toroidal moment develop that are not present in the centered structure. Practically, the $\alpha$ parameter used in the present work (as defined above) constitutes a lower boundary of the $\alpha$ coefficients that allow the stabilization of the $90^{\circ}$ domains in each quadrant. Its numerical value is between five and seven times smaller than the first-principles-derived quartic coefficients associated with anharmonicity in bulk $\mathrm{BaTiO}_{3}$ (such quartic coefficients appear in the local mode self energy of work by Zhong et al. ${ }^{20}$ and are denoted by $\alpha$ and $\gamma$ in their work). This suggests that the value of our $\alpha$ parameter is reasonable.

To probe further, the total internal energy in the platelets was calculated as a function of the quadrant-quadrupole position, using the aforementioned Hamiltonian. This total energy was computed for a number of different rectangular platelets with different sidewall aspect ratios. Normalized energy differences between the centered quadrant core and off-centered cores as a function of the degree of off-centering and rectangular aspect ratio are shown in Fig. 6(a). Clearly, there is a transition from a single-well to a double-well potential in moving from square to rectangular platelets, reminiscent of the changes in energy profiles classically associated with bulk phase transitions in ferroelectric and ferromagnetic systems; notably, the degree of off-centering for which the energy is minimum evolves continuously to larger values as the aspect ratio increases.

Such strong parallels with conventional phase transitions prompted an investigation into the extent to which the functions shown in Fig. 6(a) could be mapped to Landau energy expansions of the form:

$$
\frac{\Delta E}{E_{0}}=A\left[1-\left(\frac{2 x}{L}\right)\right]^{2}+B\left[1-\left(1-\frac{2 x}{L}\right)\right]^{4},
$$


(a)

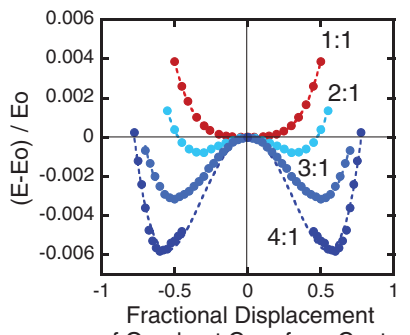

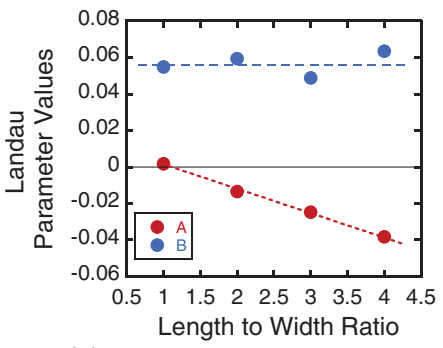

(b)
FIG. 6. (Color online) (a) Modeled normalized energy differences between states where the quadrant core is centered and where it is off-centered, given as a function of the degree of off-centering, and the aspect ratio of the sidewall dimensions in the platelets. Clearly, there is a transition from a single-well to a double-well energy function in moving from square to rectangular platelets, reminiscent of the changes in energy profiles classically associated with phase transitions. (b) Indeed, fitting these curves to a conventional Landau expansion of the form: $\frac{\Delta E}{E_{0}}=A\left[1-\left(\frac{2 x}{L}\right)\right]^{2}+B\left[1-\left(\frac{2 x}{L}\right)\right]^{4}$ (where $x$ is the distance between the quadrant core and the nearest end in the platelet, and $L$ is the platelet length) gave well-behaved scalar parameters $A$ and $B ; B$ was found to be approximately constant, and $A$ was found to change sign through the shape-induced phase transition.

where, as stated previously, $x$ is the distance between the quadrant core and the nearest end of the platelet, and $L$ is the platelet length; $E_{0}$ is the total energy when the quadrant core is positioned halfway along the length of the platelet, and $\Delta E$ is the difference between the energy calculated for the quadrant core at a particular value of off-centering and that when it is positioned halfway along the platelet length.

Figure 6(b) shows how the values of the parameters $A$ and $B$ in Eq. (1) vary as the aspect ratio of the platelets changes. For Landau expansions to be meaningful, the $A$ parameter should change sign through the phase transition, and the $B$ parameter should be constant. Clearly, both these conditions are satisfied, suggesting the more fully developed Landau free energy expansion:

$$
\begin{aligned}
\frac{\Delta E}{E_{0}}= & \frac{a}{2}\left[\left(\frac{L}{W}\right)_{C}-\left(\frac{L}{W}\right)\right]\left[1-\left(\frac{2 x}{L}\right)\right]^{2} \\
& +\frac{b}{4}\left[1-\left(\frac{2 x}{L}\right)\right]^{4},
\end{aligned}
$$

where additional parameters $a$ and $b$ are positive scalars, and $W$ is the width of the rectangular platelet; $L / W$ is then the aspect ratio of the sidewall dimensions; $\left[1-\left(\frac{2 x}{L}\right)\right]$ is the fractional degree of off-centering, as noted above, and here this acts as the effective order parameter $(Q)$ for the phase transition. Note that the point at which the transition occurs, from a centered to an off-centered core position, is dependent on the aspect ratio of the platelets, transforming at a critical value just greater than one $(\sim 1.08)$. The aspect ratio thus acts in an analogous manner to the temperature in thermally driven phase transitions. Moreover, the phase transition should be classified as second order in nature since the scalar parameter $b$ is positive.
So far, the Landau expansions, such as that in Eq. (2), have only been fitted to the total energy calculated from the effective Hamiltonian used in the Monte Carlo simulations without reference to the experimental data. To see how well the Landau energy function maps to experiment, we have plotted the predicted equilibrium value of the degree of off-centering (or equilibrium order parameter, $Q_{E}$ ) as a function of platelet aspect ratio. This has been plotted on top of the experimental data presented in Fig. 3(a). As can be seen, predictions of equilibrium behavior from the Landau expansion map extremely well to the data.

As a complementary test, we have also probed the value of the critical exponent $\beta$, taken directly from experimental data, where:

$$
\left[\left(\frac{L}{W}\right)-\left(\frac{L}{W}\right)_{C}\right]^{\beta} \propto Q_{E} .
$$

Despite scatter, a best fit $\beta$ value of 0.5 (with an $r^{2}$ parameter value of 0.7 ) was found [Fig. 3(b)], the same as that expected from mean-field modeling of the kind implied by classical Landau expansions.

\section{SUMMARY AND CONCLUSIONS}

In summary, the domain patterns that form in mesoscale $\mathrm{BaTiO}_{3}$ platelets machined from bulk single crystal using a focused ion beam (FIB) microscope have been investigated. Direct imaging using scanning transmission electron microscopy revealed that domains form into sets of four quadrants, where each quadrant is composed of a number of $90^{\circ}$ stripe domains. The spatial arrangement of these quadrants, in particular the position of the point at which they met (the quadrant core), was found to be highly sensitive to the platelet shape; in detail, the quadrant core was found to progressively migrate away from the center of the platelet as the aspect ratio of the platelet increased. Monte Carlo modeling suggested that the off-centering represented a phase transition in the potential surface experienced by the quadrant core (changing from a single- to double-well form). The Monte Carlo potentials were accurately reproduced using Landau free energy expressions, in which the degree of off-centering was used as the order parameter and the aspect ratio the thermodynamic driver. ${ }^{21}$ Predictions of equilibrium core positioning from these Landau expressions accurately mapped to the experimental observations. An apparently shape-induced second-order phase transition has therefore been identified.

\section{ACKNOWLEDGMENTS}

AS, JFS, and JMG acknowledge the Engineering and Physical Sciences Research Council and the Leverhulme Trust for funding (EP/F004869/1, EP/F001630/1, EP/H00307X/1, and F/00 203/V); LB and SP acknowledge the Office of Naval Research grants N00014-04-1-0413, N00014-08-1-0915, and N00014-07-1-0825 (DURIP), the National Science Foundation grants DMR 0701558, 0722625, and DMR-0080054 (C-SPIN), and Department of Energy grant DE-SC0002220. 
*m.gregg@qub.ac.uk

${ }^{1}$ M. E. Lines and A. M. Glass, Principles and Applications of Ferroelectrics and Related Materials (Clarendon Press, Oxford, 1977), p. 9

${ }^{2}$ J. F. Scott, Ferroelectric Memories (Springer-Verlag, Berlin Heidelberg, 2000), p. 2.

${ }^{3}$ A. F. Devonshire, Phil. Mag. 40, 1040 (1949).

${ }^{4}$ V. L. Ginzburg, J. Exp. Theor. Phys. SSSR 15, 739 (1945) (in Russian).

${ }^{5}$ L. D. Landau and E. M. Lifshitz, Statistical Physics Part 1, Vol. 5, 3rd ed. (Butterworth-Heinemann, Oxford, 1994), p. 446.

${ }^{6}$ I. Naumov, L. Bellaiche, and H. Fu, Nature 432, 737 (2004).

${ }^{7}$ H. Fu and L. Bellaiche, Phys. Rev. Lett. 91, 257601 (2003).

${ }^{8}$ S. Prosandeev, I. Ponomareva, I. Naumov, I. Kornev, and L. Bellaiche, J. Phys.: Condens. Matter. 20, 193201 (2008).

${ }^{9}$ D. D. Fong, G. B. Stephenson, S. K. Streiffer, J. A. Eastman, O. Auciello, P. H. Fuoss, and C. Thompson, Science 304, 1650 (2004).

${ }^{10}$ S. K. Streiffer, J. A. Eastman, D. D. Fong, C. Thompson, A. Munkholm, M. V. R. Ramana-Murty, O. Auciello, G. R. Bai, and G. B. Stephenson, Phys. Rev. Lett. 89, 067601 (2002).

${ }^{11}$ J. Junquera and P. Ghosez, Nature 422, 506 (2003).

${ }^{12}$ A. Schilling, T. B. Adams, R. M. Bowman, J. M. Gregg, G. Catalan, and J. F. Scott, Phys. Rev. B 74, 024115 (2006).

${ }^{13}$ A. Schilling, R. M. Bowman, G. Catalan, J. F. Scott, and J. M. Gregg, Nano Lett. 7, 3787 (2007).

${ }^{14}$ A. Schilling, D. Byrne, G. Catalan, K. G. Webber, Y. A. Genenko, G. S. Wu, J. F. Scott, and J. M. Gregg, Nano Lett. 9, 3359 (2009).
${ }^{15}$ P. W. Forsbergh, Phys. Rev. 76, 1187 (1949).

${ }^{16}$ L. W. Chang, M. McMillen, F. D. Morrison, J. F. Scott, and J. M. Gregg, Appl. Phys. Lett. 93, 132904 (2008).

${ }^{17}$ W. Swiech, G. H. Fecher, C. Ziethen, O. Schmidt, G. Schonhense, K. Grzelakowski, C. M. Schneider, R. Fromter, and H. P. Oepen, J. Kirschner J. of Electr. Spectroscopy and Rel. Phenom. 84, 171 (1997).

${ }^{18}$ N. Balke, S. Choudhury, S. Jesse, M. Huijben, Y. H. Chu, A. P. Baddorf, L. Q. Chen, R. Ramesh, and S. V. Kalinin, Nat. Nano. 4, 868 (2009).

${ }^{19}$ L. J. McGilly, A. Schilling, and J. M. Gregg, Nano Lett. 10, 4200 (2010).

${ }^{20}$ W. Zhong, D. Vanderbilt, and K. M. Rabe, Phys. Rev. B 52, 6301 (1995).

${ }^{21}$ We have, in addition, tried to identify a likely conjugate field for the observed domain off-centering. One possible candidate is $\nabla \times$ $D$, where $D$ is the electric displacement. $\nabla \times D$ can be rewritten as $[\varepsilon(\nabla \times E)]+[E \times(\nabla \varepsilon)]$ where $\varepsilon$ is the dielectric constant, and $E$ is the electric field. Such rewriting indicates that $\nabla \times D$ can result in a toroidal moment (since the conjugate field of an electric toroidal moment is $\nabla \times E$ ) as well as a polarization (since the conjugate field of a polarization is an electric field), consistent with the development of electrical toroidal moment and polarization implied by the Monte Carlo simulations. Moreover, $\varepsilon$ generally decreases towards the edges in an inhomogeneous anisotropic dielectric, implying that in our geometry the second term of $\nabla \times D$ could produce a displacement of the quadrant core along the long axis of the rectangular platelets (the maximum gradient in $\varepsilon$ lies parallel to the width of the platelet). 